rowers and nine eumenorrhoeic controls. ${ }^{8}$ These findings are surprising because if exercise does compensate for bone loss due to amenorrhoea rowers with eumenorrhoea should have a higher lumbar bone density than eumenorrhoeic controls. Dual photon absorptiometry measures both trabecular and cortical bone with vertebral bone trabeculae contributing only $35-50 \%$ of the total. ${ }^{15}$ This and the small size of the cohort investigated may explain why Snyder $e t$ al were unable to detect differences between the three groups.

The factors that contribute to attaining peak bone mass in the mid-30s are not known. Physical activity tends to increase peak bone mass and may reduce the risk of osteoporosis in later life. Our results show that intensive exercise directed at a specific location in the skeleton (that is, to the spine in rowing) confers additional benefit to bone mineralisation at that site and confirm that if the intensity of exercise is so high that it leads to amenorrhoea these benefits can be lost.

We thank Yiannis Koutedakis and Dr David Katz for their advice and Adrienne McQueen for supervising the measurements of bone mineral density. RLW is supported by a grant from Glaxo Pharmaceuticals (UK).
1 Lanyon LE. Bone loading, exercise, and the control of bone mass: the physiological basis for the prevention of osteoporosis. Bone 1989;6:19-21. Rubin CT, Lanyon LE. Regulation of bone formation by applied dynamic loads. I Bone foint Surg [Br] 1984;66:397-402.

Nilsson BE, Westlin NE. Bone density in athletes. Clin Orthop 1971:77: 179-82.

4 Huddleston AL, Rochwell D, Kulund DN, Harrison RB. Bone mass in lifetime tennis players. FAMA 1980;244:1107-9.

5 Pirnay F, Bodeux M, Crielaard JM, Franchimont P. Bone mineral content and physical activity. Int $\mathcal{I}$ Sports Med 1987;8:331-5.

6 Williams JA, Wagner J, Wasnich R, Heilbrun L. The effect of long-distance running upon appendicular bone mineral content. Med Sci Sports Exerc 1984;16:223-7.

7 Drinkwater BL, Nilson $\mathrm{K}$, Chesnut $\mathrm{CH}$, Bremner WJ, Shainholtz S, Southworth MB. Bone mineral content of amenorrheic and eumenorrheic athletes. N Engl f Med 1984;311:277-81.

8 Snyder AC, Wenderoth MP, Johnston CC, Hui SL. Bone mineral content of elite lightweight amenorrheic oarswomen. Hum Biol 1986;58:863-9.

9 Cann CE, Genant HK. Precis̈ measurement of vertebral mineral content using computed tomography. $\mathcal{F}$ Comput Assist Tomogr 1980;4:493-500.

McCullagh P, Nelder JA. Generalised linear models. London: Chapman and Hall, 1983.

11 Royston JP. An extension of Shapiro and Wilks W test for normality to large samples. Applied Statistics 1982;31:115-24.

12 Schweder T. A simple test for a set of sums of squares. Applied Statistics $1981 ; 30: 16-21$.

13 Lindberg JS, Fears WB, Hunt MM, Powell MR, Boll D, Wade CE. Exerciseinduced amenorrhea and bone density. Ann Intern Med 1984;101:647-8.

14 Marcus R, Cann C, Madvig P, et al. Menstrual function and bone mass in elite women distance runners. Ann Intern Med 1985;102:158-63

15 Nottestad SY, Baumel JJ, Kimmel DB, Recker RR, Heaney RP. The proportion of trabecular bone in human vertebrae. Fournal of Bone and Mineral Research 1987;2:221-9.

(Accepted 26 fune 1990)

\title{
Prevalence of HIV antibody and pregnancy in Tayside, 1984-9: background to screening
}

Robert Smith, Naren B Patel, George E D Urquhart, Peter McFaul, Patrick Neven, Peter W Howie

\section{Abstract}

Objective-To determine age specific prevalence of HIV antibody, incidence of pregnancy, and likelihood of detection and correct assignment to risk category by antenatal screening of women known to be positive for HIV antibody, from 1984 to 1989.

Design-Retrospective analysis of reproductive history and risk behaviour of women positive for HIV antibody and prediction of detection by screening on the basis of blood group samples, Guthrie tests, and rubella tests.

Setting-City of Dundee, where the prevalence of HIV is high, since the appearance of HIV in 1984, predominantly among heterosexual intravenous drug users.

Patients - All (61) women known to be positive for Departments of Obstetrics and Gynaecology and Microbiology, Ninewells Hospital and Medical School, Dundee DD1 9SY Robert Smith, FRCOG, consultant obstetrician and gynaecologist

Naren B Patel, FRCOG, consultant obstetrician and gynaecologist George E D Urquhart, FRCPATH, consultant virologist Peter McFaul, MD, lecturer in obstetrics and gynaecology

Patrick Neven, MD, visiting registrar

Peter W Howie, MD, professor of obstetrics and gynaecology

\section{Correspondence to: $\operatorname{Dr} \mathrm{R}$} Smith, Ward 37/38,

Ninewells Hospital and

Medical School, Dundee,

Scotland DD1 9SY. tory, incidence of infection, geographical location, age, date of positive test result, and reproductive history.

Results-With 61 infected women the overall minimum prevalence among women within the city of Dundee was 0.67/1000 and 2.9/1000 among women in their third decade. Of the 60 women whose reproductive history was available, 35 had 57 pregnancies, $\mathbf{3 6}$ of which occurred after seroconversion was known to have taken place, representing $8.7 \%$ of the total number of affected pregnancies reported for the United Kingdom. If antenatal screening for HIV had been performed between 1984 and 1989 it could not have detected positivity for HIV antibody in $25(42 \%)$ women who had no pregnancies during this time. Among the remaining $\mathbf{3 5}$ women, screening samples taken for blood grouping could have identified a maximum of $34(57 \%)$, samples taken to check rubella susceptibility a maximum of $22(37 \%)$, and blood spots on Guthrie cards a maximum of 19
(32\%). Retesting would have occurred in 14 women 33 times with samples taken for blood grouping, but three and four women would have been tested twice using samples taken for rubella testing and Guthrie cards respectively. Anonymous screening would have been unable to determine risk category as a history of intravenous drug use was known in $47(79 \%)$ women before testing but this was increased by a further $5(8 \%)$ who admitted to it after the test result was known.

Conclusion-Interpreting the results of antenatal screening programmes will be complex and will underestimate overall prevalence of HIV antibody among women; this will be exaggerated by strategies based on anonymous testing with Guthrie cards or on samples taken for rubella testing, which do not include women who have had an earlier loss of pregnancy. Only open testing with consent will permit satisfactory attribution to risk category and eliminate problems of retesting. Monitoring areas with a high prevalence of HIV infection may warn of increasing heterosexual spread.

\section{Introduction}

HIV type I arrived in Tayside during late 1983 or early $1984,{ }^{\prime}$ as determined by retrospective analysis of stored blood samples. The virus spread rapidly among the population using intravenous drugs, with only seven of 231 positive test results among adults being attributed to homosexual or bisexual behaviour; 205 $(89 \%)$ were attributed to intravenous drug use and $61(26 \%)$ to women (Tayside Health Board. Communicable Diseases in Tayside No 12/89). This pattern of spread is similar to that in Lothian ${ }^{2}$ but differs from that in the rest of Scotland. The reason for this difference is an estimated HIV infection rate of $50 \%$ among intravenous drug users in Edinburgh and 40\% in Dundee, compared with only $2-5 \%$ in Glasgow, where the largest proportion of the Scottish population 
lives. ${ }^{3}$ Although the city of Dundee has a population corresponding to $0.3 \%$ of that of the United Kingdom, it includes $5 \%$ of women known to be positive for HIV antibody in the United Kingdom and accounts for $8 \cdot 7 \%$ of all affected pregnancies reported. ${ }^{4}$ In view of this we analysed retrospectively pregnancies occurring in women known to be antibody positive in Tayside since the arrival of the virus to assess the potential value of antenatal testing. Any method of screening should detect most positive cases and must avoid detecting the same positive subject more than once. To be valuable, antenatal screening should also distinguish between women positive for HIV antibody with and without associated high risk activity. It is therefore pertinent to assess the potential epidemiological merits of different screening strategies with consent and risk assessment against the anonymous unlinked screening of pregnant women proposed for England and Wales.

\section{Method}

According to the registrar general of Scotland, the population of Tayside in 1988 was 393748 with 174255 people living in the city of Dundee, and the city's female population by each quinquennium age range from 15 to 49 years, in order of increasing age, was projected to be $6394,6797,6784,5866,5463,5372$, and 4717.

We obtained data on the number of tests performed and the number of all women and children positive for HIV antibody detected by voluntary, clinically indicated testing from 1984 to the end of 1989 from the virology laboratory at this hospital. Case notes were obtained for women known to be infected, and data on reproductive history, including the possibility of rubella screening and Guthrie testing, drug use, age, and social category were extracted. The risk activity reported to the laboratory was reviewed against the

TABLE I - Number of tests for HIV antibody and number of positive results in women, Tayside, 1985-9 (excluding antenatal screening tests in 1988 and 1989)

\begin{tabular}{lcrrrrr}
\hline Year of test & 1985 & 1986 & 1987 & 1988 & 1989 & Total \\
\hline No tested & 51 & 349 & 553 & 412 & 323 & 1688 \\
No positive & 16 & 25 & 12 & 6 & 2 & 61
\end{tabular}

TABLE II - Number of women positive for HIV antibody in each age group and minimum prevalence per thousand women in city of Dundee

\begin{tabular}{lccccc}
\hline Age group & $18-19$ & $20-24$ & $25-29$ & $30-34$ & $35-50$ \\
\hline $\begin{array}{l}\text { No } \\
\begin{array}{l}\text { Minimum prevalence } \\
\text { (per 1000 women) }\end{array}\end{array}$ & 6 & 27 & 17 & 7 & 4 \\
\end{tabular}

TABLE III-Number of live births, abortions, and terminations in women negative for HIV antibody and of unknown antibody state and women positive for antibody, 1984-9

\begin{tabular}{lccccccc}
\hline Year & 1984 & 1985 & 1986 & 1987 & 1988 & 1989 & Total \\
\hline $\begin{array}{c}\text { Live births: } \\
\text { Women negative for HIV antibody and of unknown } \\
\text { antibody state }\end{array}$ & 5 & 3 & 1 & 1 & 0 & 0 & 10 \\
$\begin{array}{c}\text { Women known to be positive for HIV antibody during } \\
\text { pregnancy }\end{array}$ & 1 & 3 & $4 \star$ & 5 & 1 & 1 & $15^{\star}$ \\
$\begin{array}{c}\text { Abortions: } \\
\text { Women negative for HIV antibody and of unknown } \\
\text { antibody state }\end{array}$ & 1 & 0 & 1 & 0 & 1 & 0 & 3 \\
$\begin{array}{c}\text { Women known to be positive for HIV antibody during } \\
\text { pregnancy }\end{array}$ & 0 & 1 & 1 & $1+$ & 0 & 0 & 3 \\
$\begin{array}{c}\text { Terminations: } \\
\text { Women negative for HIV antibody and of unknown } \\
\text { antibody state }\end{array}$ & 2 & 0 & 6 & 0 & 0 & 0 & 8 \\
$\begin{array}{c}\text { Women known to be positive for HIV antibody during } \\
\text { pregnancy }\end{array}$ & 0 & 2 & 7 & 3 & 3 & 3 & 18 \\
$\begin{array}{c}\text { All pregnancies and live births: } \\
\text { Women negative for HIV antibody and of unknown } \\
\text { antibody state }\end{array}$ & 8 & 3 & 8 & 1 & 1 & 0 & 21 \\
$\begin{array}{c}\text { Women known to be positive for HIV antibody during } \\
\text { pregnancy }\end{array}$ & 1 & 6 & $12^{\star}$ & 9 & 4 & 4 & $36^{\star}$
\end{tabular}

*Includes one set of twins.

†Includes one ectopic pregnancy. information available in the case records, particularly with regard to an unreported history of intravenous drug misuse.

In two cases we assumed that a previous pregnancy was in an infected woman when the infant and mother were subsequently tested and found to be positive for HIV antibody. In a further two cases positive results on cord blood testing led to subsequent maternal testing because of risk activity of the mother.

\section{Results}

Sixty one women were identified as positive for HIV antibody during 1984-9. For one woman information was available only from the laboratory request form because her case notes could not be traced. The largest number of new cases $(n=25)$ was detected in 1986, with a steady fall since then, although the peak of testing occurred in 1987 (table I). Age at first testing ranged from 18 to 50 , with most women (44/61) being in their 20s (table II). All but two of these women were unemployed or had partners who were unemployed; one of the two had acquired the infection from blood products and the other from an African source. Of the 61 women, two gave addresses outside the city and no address was available for another. From the known population data the minimum overall prevalence of infection was $0 \cdot 67 / 1000$ women in Dundee city, with peak prevalence in the age bands between 20 and 30 . The largest cluster of 26 cases occurred in an area of the city with an estimated population of 20000 , and within that area the minimum prevalence was $14 / 1000$ women aged 18 to 29 .

\section{RISK ACTIVITY}

Risk activity, as initially reported to the laboratory for the 61 women, was as follows: 48 women were intravenous drug users, nine had had heterosexual contact with a high risk partner or are known to be positive for HIV antibody, one admitted to both heterosexual contact and intravenous drug use, two had had heterosexual contact with an African source, and one had received HIV infected blood products. After examining the case records risk activity could be reattributed. After learning of their HIV positive result five of the nine women who initially admitted to high risk heterosexual exposure also admitted to intravenous drug use, increasing the number in that category to 54 . All of the women who admitted to intravenous drug use were sexually active so that the route of infection could not be determined with certainty.

\section{REPRODUCTIVE HISTORY}

Of the 60 women for whom records were available, 25 (42\%) had not had a pregnancy, and of these, seven had been sterilised before 1984, five were known to be subfertile, four had no record of a previous pregnancy, and the remaining nine had had at least one pregnancy up to, but not after, 1984. Fifty seven pregnancies occurred in 35 women during 1984-9, 21 women having only one pregnancy and 14 having two or more; table III shows the outcome according to HIV state. Of 21 pregnancies that occurred before seroconversion $(n=3)$ or before HIV state was known $(n=18), 10$ $(48 \%)$ were live births, three $(14 \%)$ were spontaneous abortions, and eight $(38 \%)$ were terminations. Of the 36 pregnancies in which HIV positivity was known at the time of pregnancy $(8.7 \%$ of all affected pregnancies reported in the United Kingdom), $15(42 \%)$ were live births with one set of twins, three $(8 \%)$ were spontaneous abortions or ectopic pregnancies, and $18(50 \%)$ were terminations. Thirteen of the liveborn children were subsequently found to be positive for HIV, and 
four children born to HIV positive mothers remained to be tested.

\section{OPPORTUNITIES FOR ANTENATAL SCREENING}

Blood grouping-During 1984-9, 25 of the 60 women positive for HIV antibody did not have a pregnancy, so that the maximum number of positive cases detectable by antenatal screening was 35 (58\%). All these 35 women had their blood group tested antenatally and positive women might have been detected with this sample source, with 14 being tested more than once (range two to five, on 33 occasions). One woman was known to be negative for HIV antibody at the time of her only live birth; she seroconverted later during the study period, reducing the possible detection rate to $57 \%(34 / 60)$.

Rubella testing - If samples for testing for rubella had been used these would have been obtained only in ongoing pregnancies and terminations of first pregnancies. A maximum of $25(42 \%)$ women might therefore have been detected, and three would have been tested twice. Three of the 25 women were known to be negative for HIV antibody at the time of blood sampling for rubella testing, further reducing the maximum detection rate to $36 \%(22 / 60)$

Guthrie cards-With Guthrie cards (for screening neonates for inborn errors of metabolism) as the source material a maximum of $22(37 \%)$ women who had live births would have been screened, with four being screened more than once. Three of the women were known to be negative for HIV antibody at the time of testing, reducing the maximum detection rate by this method to $32 \%(19 / 60)$.

\section{Discussion}

The change in risk assessment in the light of a positive result for HIV antibody probably related to drug seeking behaviour and the problems surrounding testing, but once a positive result was obtained it was less important to hide intravenous drug use. Follow up tests in the possibly infected children will be required to determine whether they have become infected. The fact that women positive for HIV antibody were of lower social class might be because those with jobs and mortgages avoided the problems associated with testing or because the disease is presently confined to people in a lower social class. What seems to have happened here is that the most vulnerable drug users sharing needles rapidly became infected, and a small number of their associates became infected, as far as we can tell, by heterosexual exposure alone. If heterosexual transmission becomes the main route of spread as has happened world wide, ${ }^{6}$ increasing numbers of people may become infected by this means. These data also show that women positive for HIV antibody are predominantly in the childbearing age groups. Many were no longer likely to produce further children by the end of the study period because they were infertile or had been sterilised. Although most admitted to intravenous drug use, they were also sexually active. Over half of the positive women had a pregnancy in the six years of the study. The density of women infected with HIV in certain parts of the city is high; these women continue to have pregnancies and therefore are capable of transmitting the virus so it is highly likely that spread might occur, especially in areas with a high prevalence of infection, by heterosexual transmission from subjects initially infected. Some of those infected near the time of the introduction of the virus may be as yet unaware of their infection. The long incubation period of the disease and the possibility of increased infectivity soon after initial infection and then as symptoms develop ${ }^{2}$ dictate a long period of surveillance to assess heterosexual spread.

Screening of our antenatal population should play an important part in monitoring this possibility, and voluntary antenatal screening of women and patients having a termination of pregnancy is currently being performed in Dundee and Lothian and will soon be reported. Our results indicate that 14 to 22 women positive for HIV would or could have been detected by testing samples for rubella and that $63-77 \%$ of the 60 women positive for HIV would have been undetected but that retesting would have occurred only three times. Using Guthrie cards would have resulted in testing 22 women who had live births, detecting at most 23 positive results from 19 women, or at least 16 positive results from 13 women, but only four would have been tested twice. This strategy would have failed to detect between $63 \%$ and $77 \%$ of known infected women. Whereas by using samples for blood grouping as the screening sample all but one woman who had a pregnancy during the time of the study would have been detected at the expense of between 39 and 54 positive results. This arises because of retesting a few women (14) in successive pregnancies. Overall, $43 \%$ of the 60 women positive for HIV antibody would have been undetected. What we have been unable to assess is the effect that knowledge of antibody state had on those women who decided to have terminations or sterilisation. The alternative strategy is voluntary testing, when data on risk behaviour may be collected. Voluntary testing on a named basis may permit further risk assessment in the light of a positive test result; voluntary testing anonymously, although still permitting risk assessment, does not permit subsequent reappraisal. The importance of risk assessment has been shown by these data, with evidence of high risk behaviour in most of the positive women, but there were clinical grounds for testing. To what extent heterosexual behaviour apparently unrelated to intravenous drug use will contribute to an increase in seroprevalence remains to be seen. This method has been suggested as appropriate for areas of high prevalence whereas anonymous unlinked screening would be appropriate for areas of low prevalence. ${ }^{5}$ The problems of anonymous unlinked screening are apparent from these data even in low prevalence areas. Whether any positive results are obtained will depend on the childbearing activity of infected women. If positive results are obtained the trend over time will depend on such factors as the timing of the introduction of the infection into a particular community, because period prevalence will change with the degree of saturation of infection of any risk group, and the age group infected, because of the movement of women into and then out of their childbearing years.

1 Urquhart GED, Scott SS, Wooldridge E, et al. Human immunodeficiency viru in intravenous drug abusers in Tayside. Communicable Diseases (Scotland) Surveillance Weekly Report 1987;9:5-10.

2 Brettle RB, Bisset K, Burns S, et al. Human immunodeficiency virus and drug misuse: the Edinburgh experience. Br Med f 1987;295:421-4.

3 Kennedy D. HIV infection in intravenous drug users. AIDS Scotland. Edinburgh: HMSO, 1988:2.

4 Davidson CF, Ades AE, Hudson CN, Peckham CS. Antenatal testing for the human immunodeficiency virus. Results from the Royal College of Obstetriians and Gynaecologiss' national study of HIV infection in pregnancy. cians and Gynaecologist

5 Gill ON, Adler MW, Day NE. Monitoring the prevalence of HIV. Br Med $\mathcal{Y}$ 1989;299:1295-8.

6 World Health Organisation. Aids. Global proiections of HIV/AIDS. Weekly Epidemiological Record 1989;64:229-31.

7 Anderson RM. The epidemiology of HIV infection: variable incubation plus infectious periods and heterogeneity in sexual activity. Fournal of the Royal Statistical Society $A$ 1988;151:66-93.

(Accepted 29 fune 1990 